\title{
The Effects of Sodium Butyrate Supplementation on the Expression Levels of PGC-1a, PPARa, and UCP-1 Genes, Serum Level of GLP-1, Metabolic Parameters, and Anthropometric Indices in Obese Individuals on Weight-Loss Diet: A Study Protocol for a Triple-Blind, Randomized, Placebo-Controlled Clinical Trial
}

SeyedAhmad Hosseini ( $\sim$ seyedahmadhosseini@yahoo.com )

Ahvaz Jundishapur University of Medical Sciences: Ahvaz Jondishapour University of Medical Sciences Parichehr Amiri

Ahvaz Jundishapur University of Medical Sciences: Ahvaz Jondishapour University of Medical Sciences Neda Roshanravan

Tabriz Medical University: Tabriz University of Medical Sciences

Maryam Saghafi-AsI

Tabriz Medical University: Tabriz University of Medical Sciences

Mitra Tootoonchian

Tabriz Medical University: Tabriz University of Medical Sciences

\section{Research Article}

Keywords: Sodium Butyrate, Obesity, Energy metabolism genes, Appetite

Posted Date: February 1st, 2022

DOI: https://doi.org/10.21203/rs.3.rs-1056677/v1

License: (1) This work is licensed under a Creative Commons Attribution 4.0 International License.

Read Full License 


\section{Abstract}

\section{Background}

Obesity is a multifaceted disease characterized by an abnormal accumulation of adipose tissue. Growing evidence has proposed microbiota-derived metabolites as a potential factor in the pathophysiology of obesity and related metabolic conditions over the last decade. As one of the essential metabolites, Butyrate affects several host cellular mechanisms related to appetite sensations and weight control. However, the effects of Butyrate on obesity in humans have yet to be studied. Thus, the present study was aimed to evaluate the effects of Sodium Butyrate (SB) supplementation on the expression levels of peroxisome proliferator activated-receptor (PPAR) gamma coactivator -1a (PGC-1a), PPARa and uncoupling protein 1 (UCP1) genes, serum level of glucagon-like peptide (GLP1), metabolic parameters, as well as anthropometric indices in obese individuals on weight-loss diet.

\section{Methods}

This triple-blind RCT will include 50 eligible obese subjects aged between 18-60 years. Participants will be randomly assigned into two groups: 8 weeks of SB (600 mg/day) + hypo-caloric diet or placebo (600 $\mathrm{mg} /$ day) + hypo-caloric diet. At weeks 0 and 8, distinct objectives will be pursued: 1) PGC-1a, PPARa, and UCP1 genes expression will be evaluated by Real-time polymerase chain reaction 2) Biochemical parameters will be assayed using enzymatic methods, 3) Insulin and GLP1 serum level will be assessed by enzyme-linked immunosorbent assay kit.

\section{Discussion}

New evidence from this trial may help fill the knowledge gap in this realm and facilitate multi-center clinical trials with a substantially larger sample size.

Trial registration: Iranian Registry of Clinical Trials: IRCT20190303042905N2. Registered on 31 January 2021. https://en.irct.ir/trial/53968

\section{Introduction}

Obesity is caused by a positive energy imbalance when energy consumed exceeds energy expenditure [1]. Obesity plays a crucial role in the genesis of many chronic disorders, including cardiovascular disease, type 2 diabetes mellitus (T2DM), rheumatoid arthritis, and various cancers, causing complications such as glucose intolerance, insulin resistance, systemic inflammation, hyperlipidemia, and hypertension [2]. The world health organization (WHO) estimates that the global prevalence of obesity has tripled since 1975. If current trends continue, by 2025, there will be 2.7 billion overweight people and more than 1 billion obese people [3]. This demonstrates that obesity has steadily increased over the last few decades and is now a global health concern [1]. 
Obesity is a multifactorial disease in which, in addition to heredity, environmental, social, physiological, and metabolic factors all play an essential role in its occurrence [4]. The appetite of obese people usually increases, while their energy expenditure decreases, owing to insufficient physical activity and brown adipose tissue dysfunction (BAT) [5].

BAT contributes significantly to energy expenditure by burning triglycerides [6] and glucose in humans [7]. In recent years, research on the relation of intestinal microbiota with obesity and epigenetic modifications have remarkably grown. The metabolic functions of the intestinal microbiota are the fermentation of dietary fiber and the production of short-chain fatty acids (SCFAs) [8]. In the lumen of the colon, the significant SCFAs are Acetate (C2), Propionate (C3), and Butyrate (C4) in a 1: 1: 3 molar ratio. Increased bacterial fermentation of SCFAs contributes to the regulation of systemic energy by reducing hepatic production of glucose and lipids $[9,10]$.

Animal models of metabolic diseases have reported that among all SCFAs, Butyrate has the highest antiobesity activity. There are several mechanisms through which Butyrate works, including preventing weight gain caused by a high-fat diet (HFD), reducing serum TG, total cholesterol, glucose, insulin resistance (IR), and improving hepatic steatosis [11]. Firmicutes and Bacteroides are the two main types of butyrate-producing bacteria; previous research has shown that these bacteria are reduced in obese people [12]. On the other hand, a study showed that butyrate-producing bacteria are higher in lean people, and when these bacteria are transferred to the intestines of people with metabolic syndrome (MetS), their IR status improves [13].

Treatment of obesity and obesity-related disturbances depends mainly on diet, exercise, and drugs to treat specific components, such as orlistat, metformin, and statins [14]. This pharmaceutical strategy can lead to drug interactions, as people with MetS may require several medications [15]. Additionally, treating one component of MetS can harm another [14]. In this regard, Butyrate, a microbial metabolite, has recently sparked much interest as a safe supplement that could decrease weight, serum glucose, cholesterol, and blood pressure while having minimal side effects $[11,16]$. Butyrate, a histone deacetylase, could exert these effects by interfering with energy balance components, increasing energy expenditure, or decreasing energy intake, by enhancement of appetite-regulating genes or glucose and lipid metabolism pathways $[17,18]$. Furthermore, Butyrate stimulates the secretion of some gastrointestinal anorexic hormones, including the glucagon-like peptide (GLP-1) [19].

Butyrate action is linked to the activation of mitochondria and energy expenditure enhancement via activation of peroxisome proliferator activated-receptor (PPAR) gamma coactivator -1a (PGC-1a). PGC-1a is a transcriptional activator identified as a viable molecular target for dietary interventions [20]. PGC-1a regulates energy metabolism and fatty acid oxidation by expressing mitochondrial biogenesis and respiration genes by interacting with several transcription factors such as PPARa and uncoupling protein 1 (UCP1) [21]. PPARa is also expressed in various tissues involved in fat oxidation in heart, vascular and endothelial cells, and regulates the expression of genes involved in the transport and beta-oxidation of fatty acids in the mitochondria [22]. PPARa stimulates lipolysis and increases the uptake of fatty acids by 
increasing the expression of fat transporters and lipid metabolizing enzymes. Studies have also shown that the activated PPARa can improve glucose metabolism and weaken inflammatory processes [23].

Uncoupling proteins (UCPs) are protein transporters in the mitochondrial membrane which separate the oxidative metabolism pathway from ADP phosphorylation and ATP synthesis via directing protons to the mitochondrial matrix to produce heat [24]. Therefore, UCPs are candidate genes for obesity and metabolic complications associated with obesity, and measuring the expression of UCPs is a method for assessing the oxidation of fatty acids $[25,26]$. On the other hand, activating genes related to UCPs have been identified as BAT activation markers. Increasing BAT activity is also vital for energy metabolism and is considered as a key factor that affects insulin sensitivity [27].

Overall, evidence from in vivo and in vitro studies showed that Butyrate has a remarkable impact on metabolic regulation and body composition [11]. Butyrate has been strongly linked to increased energy expenditure, fatty acid oxidation, and decreased energy intake. Therefore, it appears that Butyrate can be considered a potential therapeutic agent for obesity and Mets. Hence, due to eminent anti-obesity effects of Butyrate as well as lack of human clinical trials to evaluate the effects of Butyrate in obesity, the present study was aimed to investigate the effects of Sodium Butyrate (SB) supplementation on the expression levels of PGC-1a, PPARa, and UCP1 genes, serum level of GLP-1, metabolic parameters, and anthropometric indices in obese individuals.

\section{Methods}

\section{Study design and setting}

A triple-blind, parallel-group, single-center RCT will be conducted. The proposed RCT will take place in the Faculty of Nutrition and Food Sciences, Tabriz University of Medical Sciences for eight weeks to see how daily $600 \mathrm{mg}$ SB supplementation affects obese people. Figure 1 depicts the study flow chart.

\section{Participants}

The study will involve 50 obese individuals. Participants will be both male and female aged 18 to 60 years old; body mass index (BMI) of 30 to $40 \mathrm{~kg} / \mathrm{m} 2$; Non-inclusion criteria will be included: lack of interest in continuing the study; having diabetes, kidney diseases, heart failure, rheumatic diseases, cancers, liver failure, gastrointestinal disorders; history of obesity surgery; following a weight-loss diet or taking any supplements or weight-loss drugs in the last 6 months; smoking; pregnancy, lactation, or menopause.

\section{Registries and Ethics}

Eligible participants will receive the study protocol and sign an informed consent form. This protocol was approved by Ethics Committee of Ahvaz Jundishapur University of Medical Sciences (approval number: IR.AJUMS.REC.1399.845). The research was also registered at the Iranian Registry of Clinical Trials (IRCT20190303042905N2). 


\section{Sample size}

The sample size was estimated based on serum changes of GLP-1 [28], 95 percent confidence interval, and 80 percent power $(\alpha=0.05$ and $\beta=0.2)$. Furthermore, the mean and standard deviation [29] of GLP-1 levels in the study mentioned above were, as follows: $\Delta \mu=17.84$; SD1 $=17.93 ;$ SD2 $=22.49$. A $20 \%$ dropout rate was considered. Lastly, 25 subjects were considered for each group. Individuals who fulfilled the eligibility criteria will be included in the RCT.

\section{Blinding and randomization of participants}

All participants will be randomly assigned into two groups (intervention or placebo), stratified by age (two age groups: 18-39 years and 40-60 years) and gender (male and female). The intervention group will receive SB supplementation + hypo-caloric diet $(n=25)$ and placebo group will receive placebo capsule + hypo-caloric diet $(n=25)$. The random block design will be performed by a third person in blocks of two using Random Allocation Software (RAS). Random numbers will be labeled as SB or placebo bottles, and even or odd numbers will be randomly assigned to the study groups. To maintain blindness, instead of $A$ or B, the third person will use specific codes. The bottles will be similar in appearance and weight to achieve blinding. The treatment allocation will be concealed from participants, researchers, and outcome assessors.

\section{Intervention}

The intervention group will receive one SB capsule $(600 \mathrm{mg})$ with breakfast for 60 days. The dosage of SB supplementation was determined, based on the previous study [28]. SB supplement (550 mg Butyrate, $50 \mathrm{mg}$ hydroxypropyl methylcellulose, medium-chain triglycerides (MCT), sodium hydroxide, and purified water) will be provided by Body Bio Company (BodyBio, USA). The control group will receive the same amount of placebo capsules for 60 days. Pharmacy Faculty of Tabriz University of Medical Sciences will provide placebo capsules (Tabriz, Iran, containing $600 \mathrm{mg}$ of carboxymethyl cellulose). Size and color of SB capsules and placebo capsules are exactly similar. Both groups will receive SB and placebo capsules every 30 days. The remaining capsules returned by participants will be counted to determine adherence to the supplements in all patients. Adherence will be defined as consuming 90 percent of the supplements.

An expert dietician will design an individualized hypo-caloric diet for each participant. The Mifflin St. Jeor equation will be used to calculate total energy expenditure [30] requirements. Then, $500 \mathrm{kcals}$ of the estimated energy needs will be deducted. The hypo-caloric diet composition will consist of 10-15 percent protein, 25-30 percent fat, and 55-60 percent carbohydrate. Compliance with diet will be checked weekly by phone calls as well as a 3-day food record at the onset and end-point of the study. Figure 2 depicts the schedule for the present study.

\section{Evaluation of diet, physical activity, and appetite}

A checklist will be filled out at baseline, which includes demographic data, medical and drug history. For evaluation of dietary intake, the participants will be asked to keep a 3-day (one weekend and two 
weekdays) food record. Energy and nutrients analysis will be performed using Nutritionist IV (Hearst Corporation, USA). Physical activity level (PAL) will be calculated by the International Physical Activity Questionnaire short form (IPAQ-SF) [31]. To assess appetite, the participants will complete a visual analog scale (VAS) at baseline and end of study [32].

\section{Anthropometric Assessments}

Body weight will be measured for subjects wearing minimal clothing (Seca scale) and height will be measured by a wall-mounted meter. To calculate BMl, weight $(\mathrm{kg})$ will be divided by height squared $(\mathrm{m} 2)$. An accurate measurement of waist circumference will be taken, using a tape meter at the midpoint between the lowest rib and iliac crest at the end of normal expiration. Hip circumference (HC) will be measured at its widest point over the greatest trochanters. Waist/hip ratio (WHR) will also be calculated. Fat-free mass $(\mathrm{kg})$, fat-free mass percent, body fat mass $(\mathrm{Kg})$, and body fat percent, as well as visceral fat will be estimated through MC- 780 TANITA body analyzer (Tanita, Amsterdam, The Netherlands).

\section{Biochemical assays}

Five $\mathrm{mL}$ venous blood sample will be taken after a 12-h overnight fasting in vacuumed gel separator tubes at baseline and end of the study. Blood samples will be centrifuged at 3000 RPM for 5 minutes to extract the serum. Serum aliquots will be frozen $\left(-80^{\circ} \mathrm{C}\right)$ to measure GLP1 $(\mu \mathrm{g} / \mathrm{mL})$ and insulin $(\mu \mathrm{U} / \mathrm{mL})$ using Enzyme-linked immunosorbent assay (ELISA) kits.

Fasting blood sugar (FBS) (mg/dL), triglyceride (TG), total cholesterol (TC), high-density lipoprotein cholesterol (HDL-C), aspartate transaminase (AST), alanine transferase (ALT), and Alkaline phosphatase (ALP) will be measured by enzymatic colorimetric kits. The Friedewald equation will be used to compute low-density lipoprotein cholesterol (LDL-C) concentrations. Calculation of the homeostatic model assessment for IR (HOMA-IR) will be done using the following formula: HOMA-IR = [fasting insulin $(\mu \mathrm{lU} / \mathrm{mL}) \times$ fasting glucose $(\mathrm{mg} / \mathrm{dL})] / 405[33]$.

\section{Gene expression assay}

Five $\mathrm{mL}$ of blood samples will be collected in EDTA/K2 tubes. Peripheral blood mononuclear cells (PBMCs) will be isolated using Ficoll-Hypaque density gradient centrifugation. Total RNA extraction will be done using Trizol reagent, according the manufacturer's instruction. After designing primers, the mRNA gene expression of PGC-1a, PPARa, and UCP1 genes will be applied using Real-time polymerase chain reaction (Real time-PCR). In Real time-PCR tests, the housekeeping gene will be $\beta$-actin. The fold change of gene expressions will be calculated based on the Livak formula $\left(2^{-\Delta \Delta C t}\right)[6]$.

\section{Outcomes}

Primary outcomes will be anthropometrics indices, serum levels of GLP1, gene expression levels of PGC1a, PPARa, and UCP1. Secondary outcomes consist of lipid profile (serum levels of TG, total cholesterol, HDL-C, and LDL-C), FBS, fasting plasma insulin level, HOMA-IR, ALT, AST, and ALP. 


\section{Statistical analysis}

Data analysis of the present RCT will be conducted on an intention to treat (ITT) participants. The data will be checked for accuracy and completeness at random intervals. The data will be expressed as means \pm SD. For each variable, the percentage change will be calculated, as follows: $[(E-B) / B \times 100]$; $E$ is the end value and $B$ is the baseline value of the variable.

The Kolmogorov-Smirnov test will be used to examine the normality of the data. The paired sample t-test and Independent sample t-test will be performed for the comparison of parametric data within and between groups, respectively. For non-parametric variables, the Mann-Whitney test and Wilcoxon signed rank test will be applied. Confounding factors including baseline values will be controlled using the analysis of covariance (ANCOVA) test. IBM SPSS software (version 21) (Armonk, NY, USA) will be used to analyze the data. A P-value less than 0.05 will be considered a statistically significant.

\section{Adverse effects, safety, and data monitoring}

SB supplementation at dosage of $600 \mathrm{mg} /$ day is not known to have any side effect [11]. A Data Monitoring Committee (DMC) will monitor the outcome of the RCT. Furthermore, the report of potential side effects will also be forwarded to the Ethics Committee of Ahvaz University of Medical Sciences.

\section{Discussion}

According to the recent animal studies, microbiota-associated metabolites may play an imperative role in the weight management and insulin-related issues. Based on several health benefits of Butyrate and the lack of adequate human studies, RCTs are required to evaluate the effects of SB supplementation on markers of metabolic pathway and anthropometric indices in obese subjects. The strengths of this tripleblind randomized controlled clinical trial are the evaluation of the effects of SB supplementation and individualized hypo-caloric diet plan on obese subjects. We expect that the outcomes of this trial, whether positive or negative, will fill the gap in the literature and facilitate the development of multi-center clinical trials with a substantially larger sample size.

\section{Trial status}

Recruitment for this trial has begun at June 2021 and is expected to be completed by November 2021 (Now, it is underway).

\section{Abbreviations}

\section{BAT}

Brown adipose tissue

BMI

Body mass index

ELISA 
Enzyme-linked immunosorbent assay

GLP1

glucagon-like peptide1

HDL-C

High-density lipoprotein cholesterol

HFD

high fat diet

HOMA-IR

Homeostasis model assessment of insulin resistance

ITT

Intention to treat

$\mathrm{IR}$

Insulin resistance

MetS

metabolic syndrome

LDL-C

Low-density lipoprotein cholesterol

PPAR

peroxisome proliferator activated-receptor

PGC-1a

peroxisome proliferator activated receptor gamma coactivator-1a

QPCR

Quantitative polymerase chain reaction

RCT

Randomized clinical trial

SB

Sodium Butyrate

SCFA

Short-chain fatty acid

SD

Standard deviation

T2DM

Type 2 diabetes mellitus

TC

Total cholesterol

TG

Triglycerides

UCP1

uncoupling protein 1

WC 
Waist circumference

WHR

Waist-to-hip ratio

\section{Declarations}

\section{Ethics approval and consent to participate}

This trial was approved by the Ethics Committee of Ahvaz Jundishapur University of Medical Sciences (approval number: IR.AJUMS.REC.1399.845). Informed consent will be obtained from all study participants prior to the study onset.

\section{Consent for publication}

Not applicable.

\section{Availability of data and materials}

The dataset generated during the current study will be available via the corresponding author upon reasonable request. Results and findings of the study will be released through publications in scientific literature and conference presentations.

\section{Competing interest}

The authors declare no competing interests.

\section{Funding}

The present study is funded by Ahvaz Jundishapur University of Medical Sciences, Ahvaz, Iran. The funding body did not have any involvement in the study design and will not have any role in the execution, data collection, data analysis, and interpretation, or preparation and publishing of the manuscript.

\section{Author Contributions}

Study design: PA and SAH. Methodology: PA, NR and MSA. Statistical plan: PA, SAH. Coordination of the study implementation: PA, MSA and NR. Data collection: PA and MT. Manuscript preparation: PA. Review and editing: MSA, SAH and PA.

All named authors have read and approved the final manuscript, adhere to the authorship guidelines of journal and have agreed to publication.

\section{Acknowledgment}


We would like to acknowledge the staff of the Faculty of Nutrition and Food Sciences, Tabriz Medical University of Medical sciences and Ahvaz Jundishapur University of Medical Sciences for their support and assistance with the trial best performance.

\section{References}

1. Blüher, M., Obesity: global epidemiology and pathogenesis. Nature Reviews Endocrinology, 2019. 15(5): p. 288-298.

2. Ortega, F.B., C.J. Lavie, and S.N. Blair, Obesity and cardiovascular disease. Circulation research, 2016. 118(11): p. 1752-1770.

3. Nittari, G., et al., Fighting obesity in children from European World Health Organization member states. Epidemiological data, medical-social aspects, and prevention programs. Clin Ter, 2019. 170(3): p. e223-230.

4. Grundy, S.M., Metabolic syndrome update. Trends in cardiovascular medicine, 2016. 26(4): p. 364373.

5. Labbé, S.M., et al., Hypothalamic control of brown adipose tissue thermogenesis. Frontiers in systems neuroscience, 2015. 9: p. 150.

6. LIVAKKJ, S., Analysisofrela凶 tivegeneexpressiondatausingreal $\otimes$ timequantitative PCRandthe2 ( $\mathrm{Q}$ DeltaDeltaC (T)) method. $\| \nabla \nabla \nabla \nabla \nabla .43(1)$ : p. 79.

7. Cypess, A.M. and C.R. Kahn, Brown fat as a therapy for obesity and diabetes. Current opinion in endocrinology, diabetes, and obesity, 2010. 17(2): p. 143.

8. Pascale, A., et al., Microbiota and metabolic diseases. Endocrine, 2018. 61(3): p. 357-371.

9. Fan, Y. and O. Pedersen, Gut microbiota in human metabolic health and disease. Nature Reviews Microbiology, 2020: p. 1-17.

10. Sukkar, A.H., et al., Regulation of energy expenditure and substrate oxidation by short-chain fatty acids. Journal of Endocrinology, 2019. 242(2): p. R1-R8.

11. Bridgeman, S.C., et al., Butyrate, generated by gut microbiota, and its therapeutic role in metabolic syndrome. Pharmacological Research, 2020: p. 105174.

12. Den Besten, G., et al., The role of short-chain fatty acids in the interplay between diet, gut microbiota, and host energy metabolism. Journal of lipid research, 2013. 54(9): p. 2325-2340.

13. Vrieze, A., et al., Transfer of intestinal microbiota from lean donors increases insulin sensitivity in individuals with metabolic syndrome. Gastroenterology, 2012. 143(4): p. 913-916. e7.

14. Apovian, C.M., W.T. Garvey, and D.H. Ryan, Challenging obesity: Patient, provider, and expert perspectives on the roles of available and emerging nonsurgical therapies. Obesity, 2015. 23: p. S1s26.

15. Kroner, B.A., Common drug pathways and interactions. Diabetes Spectrum, 2002. 15(4): p. $249-255$.

16. Roshanravan, N., et al., The effects of sodium butyrate and high-performance inulin supplementation on the promotion of gut bacterium Akkermansia muciniphila growth and alterations in miR-375 and 
KLF5 expression in type 2 diabetic patients: A randomized, double-blind, placebo-controlled trial. European journal of integrative medicine, 2018. 18: p. 1-7.

17. Donohoe, D.R., et al., The microbiome and butyrate regulate energy metabolism and autophagy in the mammalian colon. Cell metabolism, 2011. 13(5): p. 517-526.

18. SÄEMANN, M.D., et al., Anti-inflammatory effects of sodium butyrate on human monocytes: potent inhibition of IL-12 and up-regulation of IL-10 production. The FASEB Journal, 2000. 14(15): p. 23802382.

19. McNabney, S.M. and T.M. Henagan, Short chain fatty acids in the colon and peripheral tissues: a focus on butyrate, colon cancer, obesity and insulin resistance. Nutrients, 2017. 9(12): p. 1348.

20. Lagouge, M., et al., Resveratrol improves mitochondrial function and protects against metabolic disease by activating SIRT1 and PGC-1a. Cell, 2006. 127(6): p. 1109-1122.

21. Lin, J., C. Handschin, and B.M. Spiegelman, Metabolic control through the PGC-1 family of transcription coactivators. Cell metabolism, 2005. 1(6): p. 361-370.

22. Yamauchi, T., et al., Adiponectin stimulates glucose utilization and fatty-acid oxidation by activating AMP-activated protein kinase. Nature medicine, 2002. 8(11): p. 1288-1295.

23. Gao, Z., et al., Butyrate improves insulin sensitivity and increases energy expenditure in mice. Diabetes, 2009. 58(7): p. 1509-1517.

24. Park, J.-S., et al., Anti-inflammatory effects of short chain fatty acids in IFN- $\gamma$-stimulated RAW 264.7 murine macrophage cells: Involvement of NF-KB and ERK signaling pathways. International immunopharmacology, 2007. 7(1): p. 70-77.

25. de Oliveira, M.S., et al., The association of uncoupling proteins 1, 2, and 3 with weight loss variability after bariatric surgery: a systematic review. Surgery for Obesity and Related Diseases, 2020.

26. Margaryan, S., et al., The mRNA expression levels of uncoupling proteins 1 and 2 in mononuclear cells from patients with metabolic disorders: obesity and type 2 diabetes mellitus. Advances in Hygiene \& Experimental Medicine/Postepy Higieny i Medycyny Doswiadczalnej, 2017. 71.

27. Wankhade, U.D., et al., Novel browning agents, mechanisms, and therapeutic potentials of brown adipose tissue. BioMed research international, 2016. 2016.

28. Roshanravan, N., et al., Effect of butyrate and inulin supplementation on glycemic status, lipid profile and glucagon-like peptide 1 level in patients with type 2 diabetes: a randomized double-blind, placebo-controlled trial. Hormone and metabolic research, 2017. 49(11): p. 886-891.

29. Bouter, K., et al., Differential metabolic effects of oral butyrate treatment in lean versus metabolic syndrome subjects. Clinical and translational gastroenterology, 2018. 9(5).

30. Foley, H.M. and A.E. Steel, Adverse events associated with oral administration of melatonin: A critical systematic review of clinical evidence. Complementary therapies in medicine, 2019. 42: p. 65-81.

31. Vasheghani-Farahani, A., et al., The Persian, last 7-day, long form of the International Physical Activity Questionnaire: translation and validation study. Asian journal of sports medicine, 2011. 2(2): p. 106. 
32. Mohammadi, M., et al., The Characteristics, Reliability and Validity of the Persian Version of Simplified Nutritional Appetite Questionnaire (SNAQ). The journal of nutrition, health \& aging, 2019. 23(9): p. 837-842.

33. Antuna-Puente, B., et al., HOMA or QUICKI: is it useful to test the reproducibility of formulas? Diabetes \& metabolism, 2008. 34(3): p. 294-296.

Figures 
Recruiting 50 eligible subject based on inclusion and exclusion criteria

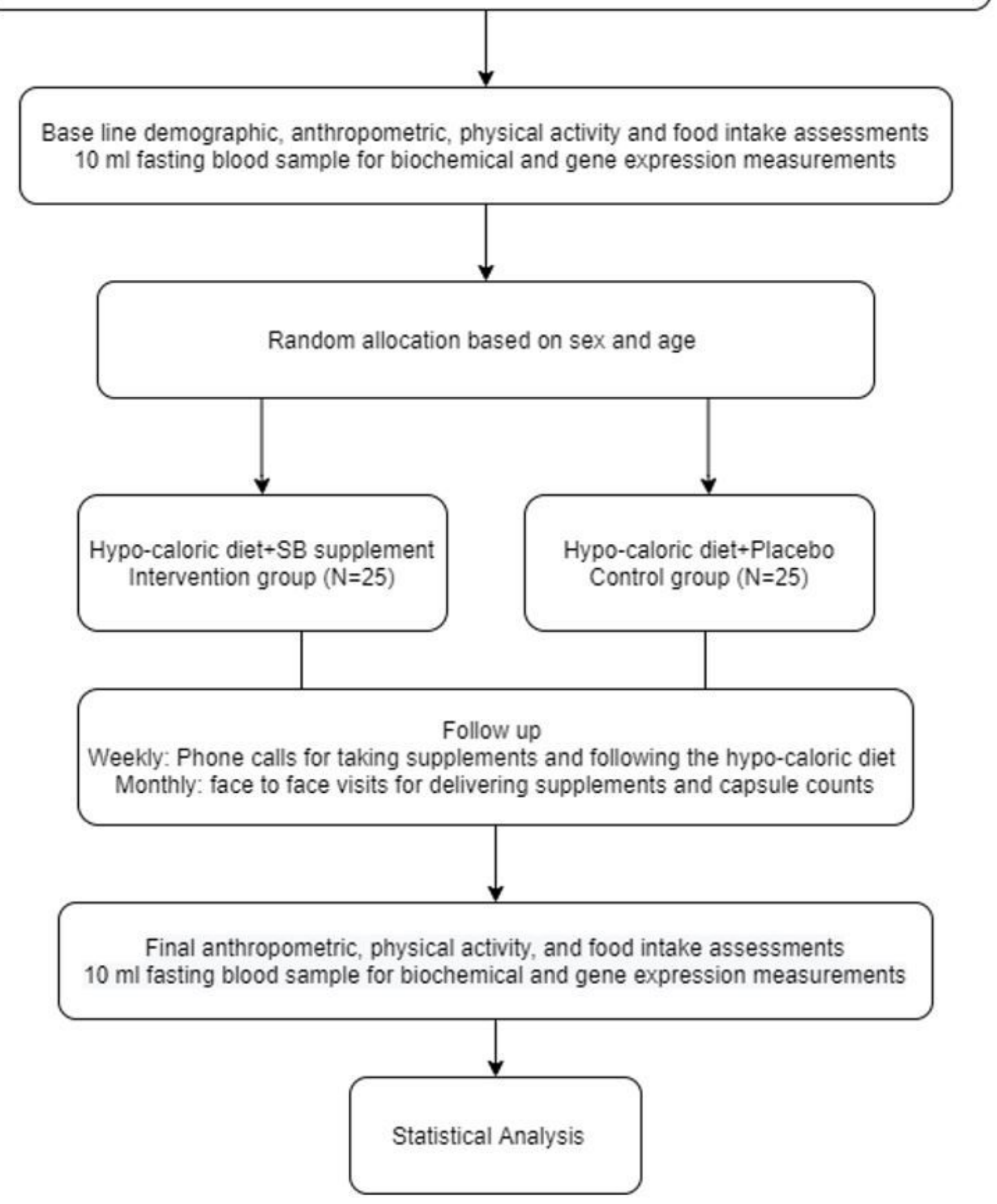

\section{Figure 1}

Study flow chart 


\begin{tabular}{|c|c|c|c|c|c|c|c|c|}
\hline \multirow[b]{3}{*}{ TIMEPOINT ** } & \multicolumn{8}{|c|}{ STUDY Period } \\
\hline & Enrolment & Allocation & Post-a & locati & & & & Close-out \\
\hline & -1 week & week0 & $\begin{array}{c}\text { Week } \\
1\end{array}$ & $\begin{array}{c}\text { Week } \\
2\end{array}$ & $\begin{array}{c}\text { Week } \\
4\end{array}$ & $\begin{array}{c}\text { Week } \\
6\end{array}$ & $\begin{array}{c}\text { Week } \\
8\end{array}$ & $\begin{array}{l}\text { End of } \\
\text { week } 8\end{array}$ \\
\hline \multicolumn{9}{|l|}{ ENROLMENT: } \\
\hline Eligibility screen & $\mathrm{X}$ & & & & & & & \\
\hline Informed consent & $\mathrm{X}$ & & & & & & & \\
\hline Allocation & & $\mathrm{X}$ & & & & & & \\
\hline \multicolumn{9}{|l|}{ INTERVENTIONS: } \\
\hline \multicolumn{9}{|l|}{ SB+hypo-caloric diet } \\
\hline \multicolumn{9}{|l|}{ ASSESSMENTS: } \\
\hline [Primary outcomes] & & $\mathrm{X}$ & & & & & & $\mathrm{X}$ \\
\hline [Secondary outcomes] & & $\mathrm{X}$ & & & & & & $\mathrm{X}$ \\
\hline
\end{tabular}

Figure 2

SPIRIT Figure: Schedule for enrollment, intervention, and assessment

\section{Supplementary Files}

This is a list of supplementary files associated with this preprint. Click to download.

- SupplementaryFile1SPIRITChecklist.doc 